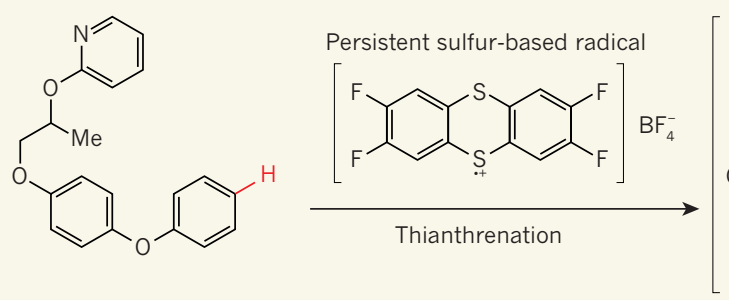

Figure 1 | A method for the site-selective modification of complex molecules. Berger et al. ${ }^{1}$ report that a chemical species known as a persistent sulfur-based radical, generated in situ, reacts with structurally complex molecules at just one carbon-hydrogen $(\mathrm{C}-\mathrm{H})$ bond (shown in red in this example; other $\mathrm{C}-\mathrm{H}$ bonds in the molecule are not shown). Which $\mathrm{C}-\mathrm{H}$ bond reacts depends on the electronic and structural properties of the
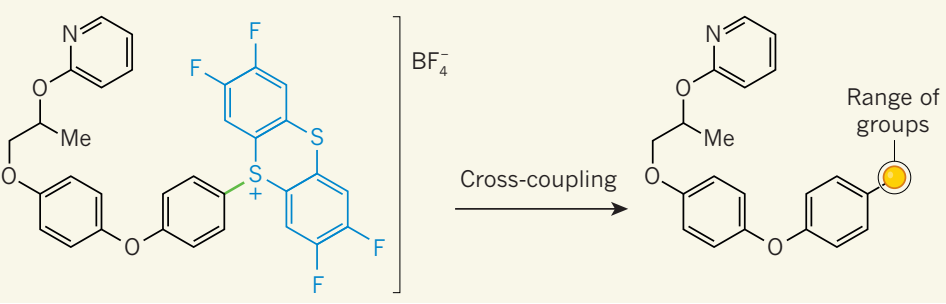

reactant. The products are compounds that bear a thianthrenium group (blue; the dot on the lower sulfur atom represents an unpaired electron). A carbon-sulfur bond (green) in these products can take part in a variety of metal-based cross-coupling reactions, thus allowing a wide range of chemical groups to be attached at the carbon atom in the original $\mathrm{C}-\mathrm{H}$ bond. $\mathrm{Me}$, methyl group.
But despite impressive advances, metalcatalysed $\mathrm{C}-\mathrm{H}$ functionalizations are not yet applicable to as broad a range of compounds as are cross-couplings, and they generally have site-selectivity issues similar to those of halogen-installing reactions.

Berger et al. now describe a development that could help to address the issues of molecular decoration. Inspired by research from almost 50 years $\mathrm{ago}^{4}$, the authors report a highly selective method for replacing the hydrogen of a $\mathrm{C}-\mathrm{H}$ bond with a group called a thianthrenium, thereby generating a new $\mathrm{C}-\mathrm{S}$ bond (Fig. 1). The thianthrenium group then serves as a halogen proxy for a broad array of cross-coupling reactions.

The first step of the process - the thianthrenation step - is strikingly siteselective. This is because the thianthrenium group derives from a species known as a persistent sulfur-based radical ${ }^{5,6}$; the use of this radical promotes a reaction that discriminates clearly between the many $\mathrm{C}-\mathrm{H}$ bonds in a given molecule. Berger et al. demonstrate that this selectivity holds across a wide range of molecules, and thereby provide data that will help chemists to work out which site is likely to react in complex molecules that have several potentially reactive $\mathrm{C}-\mathrm{H}$ bonds. Essentially, in almost all of the cases evaluated, only one $\mathrm{C}-\mathrm{H}$ bond reacts, with the selectivity dictated by the electronic and structural characteristics of each molecule.

Equally impressive is the breadth of the cross-coupling reactions that can be carried out using the thianthrenated compounds, in which the C-S bond acts as an 'activated' carbon-halogen bond ${ }^{7}$. A variety of palladiumcatalysed cross-couplings are effective and, in the most notable examples, the thianthrenium group reacts in preference to carbon-halogen bonds or other similarly reactive groups. The authors also find that their thianthrenium compounds work in other types of crosscoupling reaction, such as copper-mediated processes and 'photoredox' couplings.

About half of the molecules tested by Berger and colleagues in their reactions are pharmaceutical compounds, which demonstrates the potential value of this chemistry to medicinal chemists. Having the ability to decorate structurally complex molecules with different chemical groups is particularly useful for synthesizing analogues of biologically active compounds in drug-discovery programmes ${ }^{8}$. It is difficult to think of a comparable reaction that works for this purpose as selectively and with as broad a range of substrates as the newly reported chemistry.

Nevertheless, these reactions might not be applicable to all molecules. Some chemical groups are sensitive to the thianthrenation conditions (which are oxidizing), and might therefore take part in oxidative side reactions. Moreover, the thianthrenated compounds have not yet been shown to take part in certain widely used cross-coupling reactions (notably, amination reactions, which generate carbonnitrogen bonds). More work is needed to develop such reactions.

Finally, the site selectivity of the thianthrenation step is governed entirely by the electronic and structural properties of the reactant molecule. It will be interesting to see whether alternative $\mathrm{C}-\mathrm{H}$ sites on molecules can be selectively thianthrenated using different protocols.
In the meantime, Berger et al. have established the foundations of a compelling new approach for cross-coupling that takes advantage of $\mathrm{C}-\mathrm{H}$ functionalization. In effect, the authors have developed an adhesive for decorating molecules that makes it much easier to generate any structure that we might need.

Eric M. Ferreira is in the Department of Chemistry, University of Georgia, Athens, Georgia 30602, USA.

e-mail:emferr@uga.edu

1. Berger, F. et al. Nature 567, 223-228 (2019).

2. Wu, X.-F., Anbarasan, P., Neumann, H. \& Beller, M. Angew. Chem. Int. Edn 49, 9047-9050 (2010).

3. Chen, X., Engle, K. M., Wang, D.-H. \& Yu, J.-Q. Angew. Chem. Int. Edn 48, 5094-5115 (2009).

4. Shine, H. J. \& Silber, J. J. J. Org. Chem. 36, 2923-2926 (1971).

5. Griller, D. \& Ingold, K. U. Acc. Chem. Res. 9, 13-19 (1976).

6. Romero, K. J., Galliher, M. S., Pratt, D. A. \& Stephenson, C. R. J. Chem. Soc. Rev. 47, 7851-7866 (2018).

7. Srogl, J., Allred, G. D. \& Liebeskind, L. S. J. Am. Chem. Soc. 119, 12376-12377 (1997).

8. Cernak, T., Dykstra, K. D., Tyagarajan, S., Vachal, P. \& Krska, S. W. Chem. Soc. Rev. 45, 546-576 (2016). resistance is rising. Treating mosquitoes with drugs that target the disease-causing parasite offers another way of tackling malaria. SEE LETTER P.239

\section{JANET HEMINGWAY}

A ny long-term programme for disease control that relies on one type of intervention has a high probability of failure owing to the development of treatment resistance. Efforts to limit malaria by using insecticides to kill female mosquitoes, which transmit the disease-causing Plasmodium parasite, are no exception: mosquito resistance to insecticides is on the increase. On page 239, Paton et al. ${ }^{1}$ report a non-insecticidal intervention that stops mosquitoes from transmitting malaria and that might offer a way to reduce the reliance on insecticides alone as a means of malaria prevention.

It is estimated ${ }^{2}$ that mosquito control using bed nets impregnated with long-lasting insecticides called pyrethroids, and indoor insecticide spraying, resulted in 1.3 billion fewer 
malaria cases and 6.8 million fewer deaths between 2000 and 2015. However, despite these intensive efforts, a 2018 report $^{3}$ by the World Health Organization (WHO) states that, between 2015 and 2017, global efforts to reduce the burden of malaria stalled.

The report highlights a funding gap that has resulted in a failure to achieve universal coverage of bed nets, where needed, and an increase in resistance to antimalarial drugs and insecticides. It also mentions the limited data available on insecticide resistance, and states that the impact of pyrethroid resistance on the effectiveness of current pyrethroid-based interventions is poorly understood. Although the report says that such increasing resistance will probably reduce the efficacy of current interventions, it downplays the potential impact on the use of pyrethroid-treated bed nets, stating that the nets, which have been responsible ${ }^{4}$ for approximately $68 \%$ of the estimated reduction in malarial transmission since 2000, continue to be effective. Sadly, this optimism seems misplaced, given the evidence that the increased insecticide resistance is affecting the level of disease transmission.

Models had already predicted ${ }^{5}$ that insecticide resistance would affect malaria transmission. Even low levels of insecticide resistance would increase the incidence of malaria because of the effect of reduced mosquito mortality and lower overall community protection during the projected three-year lifespan of a bed net. A type of bed net called a PBO net that contains pyrethroid and piperonyl butoxide, a compound that enhances pyrethroid's effectiveness, would be substantially more effective than pyrethroid-only nets ${ }^{5}$. It is estimated that switching from pyrethroidonly bed nets to PBO nets would prevent up to 0.5 clinical cases of malaria per person per year in some scenarios ${ }^{5}$. Trials have demonstrated that, in insecticide-resistant areas, $\mathrm{PBO}$ nets are more effective at reducing the transmission of malaria than are pyrethroid-only nets ${ }^{6}$.

Given the urgent need to expand the range of effective interventions, Paton et al. suggest a way forward for the development of nextgeneration antimalarial bed nets. They tested whether Plasmodium falciparum, the major malaria-causing parasite in Africa, could be killed by exposing mosquitoes to the parasitekilling drugs that are used to prevent people from getting malaria. These work by targeting mitochondria - energy-generating organelles.

The authors found that the antimalarial drug atovaquone, which inhibits the mitochondrial protein cytochrome $b-$ as well as other types of cytochrome $b$ inhibitor drug - could kill parasites in a mosquito host. Exposing mosquitoes for six minutes to a glass surface coated

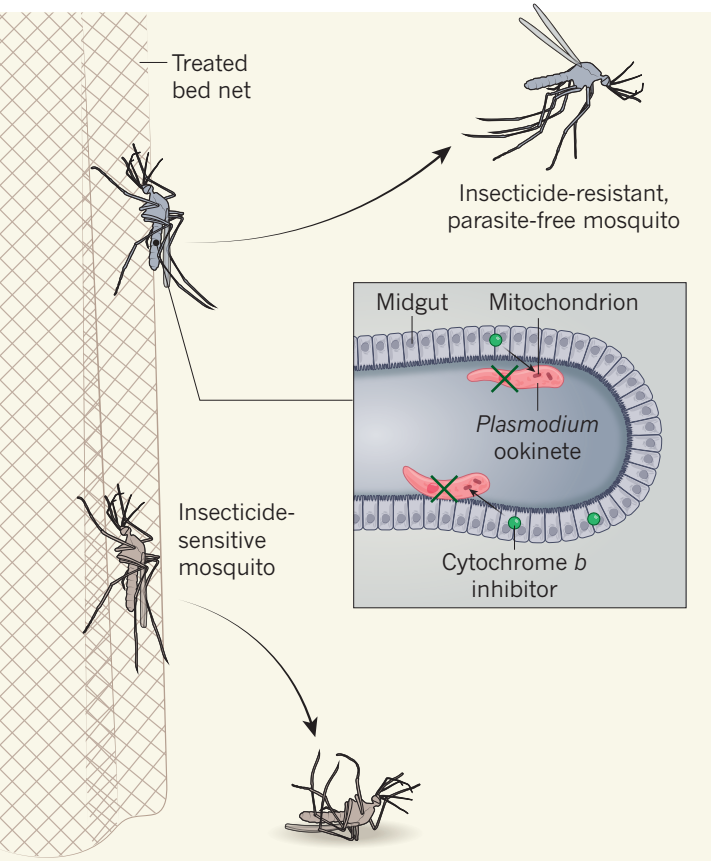

Figure 1 | How a non-insecticidal treatment might be used to block malaria transmission. Transmission of the Plasmodium parasite to humans by mosquitoes causes malaria. A standard disease-prevention approach is the treatment of bed nets with insecticides that kill mosquitoes. However, mosquito resistance to insecticides is on the rise. Paton et al. ${ }^{1}$ report that exposing mosquitoes to surfaces treated with antimalarial drugs targeting the parasite kills the parasite in its host, and they propose that bed-net treatment that combines both insecticides and such arasite-targeting compounds might aid efforts to tackle malaria. The authors studied drugs that inhibit the protein cytochrome $b$ mitochondria (the cell's energy-generating organelles) in lasmodium. They report that these drugs block the development ookinete-stage Plasmodium parasites in the mosquito midgut. illing the parasite in this way would prevent insecticide-resistant osquitoes from transmitting disease.

with low doses of the drug was enough to have an effect. This is within the time frame for which wild mosquitoes rest on bed nets. The finding is important because, after more than a century of research, and small-scale projects involving biological or environmental control, it is evident that non-insecticidal interventions do not contribute substantially to efforts to reduce malaria transmission.

Paton and colleagues suggest that antimalarial drugs could be incorporated into bed nets alongside insecticides (Fig. 1). Their modelling predicts that this would reduce the prevalence of malaria, with the size of the impact being affected by the levels of disease transmission, the number of people using nets and the degree of insecticide resistance. The increased effect of adding the antimalarial drug to insecticide-treated nets is most striking in the authors' models when bed-net usage is $70-100 \%$, underscoring the need to strive for high levels of net usage even if the compounds used to treat the nets change in the future.

Malaria cannot be sustainably controlled or eliminated without effective mosquito control. Paton and colleagues' approach fits well with the WHO's global plan for managing insecticide resistance ${ }^{7}$. This suggests that a multifaceted approach should be used, and progress will rely on efforts to reduce the cost of bringing new drugs to market and streamlining the associated development, policy and implementation steps ${ }^{8}$.

Although Paton and colleagues' approach holds promise, substantial hurdles must be overcome before a product is generated that is recommended and universally accepted by funders, countries and communities for use in control programmes. Extensive work will be needed to optimize drug development, and so produce long-lasting formulations. Moreover, assessments of the drug-manufacturing process should be undertaken, together with a drug's resistance potential and the cost and public acceptability of the approach.

The WHO has a system called prequalification, in which a product for tackling a health problem must meet certain specifications before it can be placed on a list for selection by donor organizations. In addition, countries have product-recommendation systems that consider proposed interventions and establish the burden of proof for whether a WHO recommendation is warranted for use there. Completing these steps is a difficult and lengthy process. For example, the first $\mathrm{PBO}$ nets were a market-ready product bearing a WHO interim recommendation ten years before the first WHO guidance was issued on where and when this type of net should be used ${ }^{2}$. The combined developmental, regulatory and other processes involved can create a 20 -year hiatus before a new technology enters the marketplace and is used at scale. Substantial effort and funding will therefore be needed to ensure a rapid evaluation of whether Paton and colleagues' suggested approach could have a role in reversing the current trend of rising malaria transmission.

Janet Hemingway is at the Liverpool School of Tropical Medicine, Liverpool L3 5QA, UK. e-mail: janet.hemingway@lstmed.ac.uk

1. Paton, D. G. et al. Nature 567, 239-243 (2019).

2. Killeen, G. \& Ranson, H. Lancet 391, 1551-1552 (2018).

3. World Health Organization. World Malaria Report 2018 (WHO, 2018); go.nature.com/2ssezu1

4. Bhatt, S. et al. Nature 526, 207-211 (2015).

5. Churcher, T. S., Lissenden, N., Griffin, J. T., Worrall, E. \& Ranson, H. eLife 5, e16090 (2016).

6. Protopopoff, N. \& Rowland, M. Lancet 391, 2415-2416 (2018).

7. World Health Organization. Global Plan for Insecticide Resistance Management in Malaria Vectors (WHO, 2012); go.nature.com/2tvtiyc 8. Hemingway, J. et al. Lancet 387, 1785-1788 (2016).

This article was published online on 27 February 2019. 